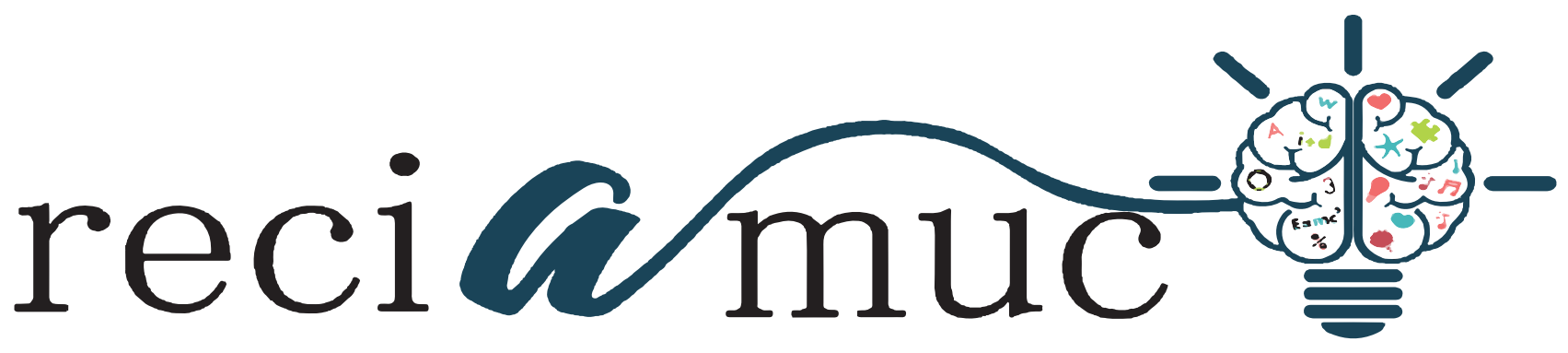

DOI: 10.26820/reciamuc/4.(4).noviembre.2020.248-254

URL: https://reciamuc.com/index.php/RECIAMUC/article/view/574

EDITORIAL: Saberes del Conocimiento

REVISTA: RECIAMUC

ISSN: 2588-0748

TIPO DE INVESTIGACIÓN: Artículo de Investigación

CÓDIGO UNESCO: 3205 Medicina Interna; 3205.01 Cardiología

PAGINAS: 248-254

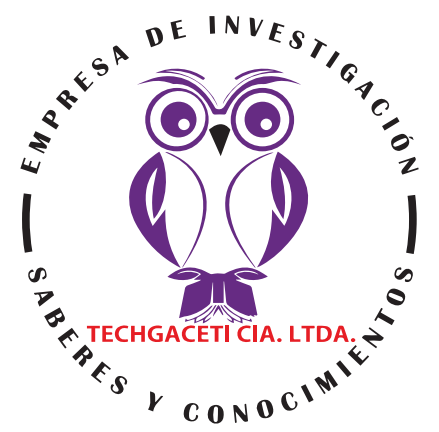

\title{
Cardiopatías asociadas al COVID-19
}

Heart disease associated with COVID-19

Doença cardíaca associada à COVID-19

\section{Stephanie Janeth Moyon Uriña'; José Miguel Villacís Nieto²; Dennis Xavier Giler Navia; Katherine Yelise Castro Caicedo ${ }^{4}$}

\author{
RECIBIDO: 18/07/2020 ACEPTADO: 20/09/2020 PUBLICADO: 30/11/2020
}

1. Médico de la Universidad de Guayaquil, Investigador Independiente; Guayaquil, Ecuador; stephaniejaneth1994@gmail. com; iD https://orcid.org/0000-0001-6357-3138

2. Médico de la Universidad de Guayaquil, Investigador Independiente; Guayaquil, Ecuador; jmvn06@hotmail.com; iD https://orcid.org/0000-0002-3259-737X

3. Médico de la Universidad de Guayaquil, Investigador Independiente; Guayaquil, Ecuador; dennisgiler@live.com; (iD https://orcid.org/0000-0003-0511-9716

4. Médico de la Universidad de Guayaquil, Investigador Independiente; Guayaquil, Ecuador; katita_1601@hotmail.com; https://orcid.org/0000-0002-6625-7939

CORRESPONDENCIA

Stephanie Janeth Moyon Uriña

stephaniejaneth1994@gmail.com

Guayaquil, Ecuador

() RECIAMUC; Editorial Saberes del Conocimiento, 2020 


\section{RESUMEN}

Vamos rumbo a cumplir ya el primer año de la detección en Wuhan, China del Sars Cov-2, y que fue declarado pandemia mundial en marzo del presente año por la Organización Mundial de la Salud, para este momento hay rebrotes de Europa y Estados Unidos, y en donde se creía la enfermedad ya había pasado, sin embargo ya hay 2 laboratorios que culminaron exitosamente las pruebas para la vacuna. Este virus que paralizo al planeta más de la mitad del año 2020, sigue generado secuelas a aquellos que lo han padecido y cobrando la vida de personas con enfermedades congénitas ya preexistentes por lo menos en poblaciones con edad de riesgo avanzadas. La metodología utilizada para esta investigación es una revisión bibliográfica, apoyada en medios electrónicos para la obtención primaria de la información, acerca de la temática de estudio. Las conclusiones de la misma es que si hay estudios derivados del primer Sars Cov que se presentó hace algunos años y confirmados por el actual, que hay personas que padecieron la enfermedad y se le generaron secuelas de índole cardiológico como arritmias cardiacas, síndromes coronarios agudos, entre otros.

Palabras clave: Covid-19, Enfermedades, Cardiológico, Arritmias, Severa.

\section{ABSTRACT}

We are on our way to completing the first year of the detection in Wuhan, China of Sars Cov-2, and which was declared a global pandemic in March of this year by the World Health Organization, at this time there are outbreaks in Europe and the United States, and where the disease was believed to have already passed, however, there are already 2 laboratories that successfully completed the tests for the vaccine. This virus that paralyzed the planet more than half of the year 2020 , continues to generate sequelae to those who have suffered it and claiming the lives of people with pre-existing congenital diseases, at least in populations with advanced risk ages. The methodology used for this research is a bibliographic review, supported by electronic means for the primary obtaining of information, about the subject of study. Its conclusions are that there are studies derived from the first Sars Cov that was presented a few years ago and confirmed by the current one, that there are people who suffered from the disease and had cardiological sequelae such as cardiac arrhythmias, acute coronary syndromes, among others.

Keywords: Covid-19, Diseases, Cardiology, Arrhythmias, Severe.

\section{RESUMO}

Estamos a caminho de completar o primeiro ano da detecção em Wuhan, China da Sars Cov-2, e que foi declarada uma pandemia global em Março deste ano pela Organização Mundial de Saúde, nesta altura há surtos na Europa e nos Estados Unidos, e onde se acreditava que a doença já tinha passado, no entanto, já existem 2 laboratórios que completaram com sucesso os testes para a vacina. Este vírus que paralisou o planeta mais de metade do ano 2020, continua a gerar sequelas para aqueles que o sofreram e a reclamar a vida de pessoas com doenças congénitas pré-existentes, pelo menos em populações com idades de risco avançadas. A metodologia utilizada para esta investigação é uma revisão bibliográfica, apoiada por meios electrónicos para a obtenção primária de informação, sobre o tema de estudo. As suas conclusões são que existem estudos derivados do primeiro Sars Cov que foi apresentado há alguns anos e confirmado pelo actual, que existem pessoas que sofreram da doença e tiveram sequelas cardiológicas como arritmias cardíacas, síndromes coronárias agudas, entre outras.

Palavras-chave: Covid-19, Doenças, Cardiologia, Arritmias, Graves. 


\section{Introducción}

No debe ser difícil asumir que, ante la no-vedad de una enfermedad contagiosa, los riesgos y las complicaciones siempre se considerarán, más aún si esa nueva patología se presenta como una epidemia de extensión o gravedad destacada. Así se ha presentado el reciente COVID-19 (Choez, Cerón, Zambrano, \& Pin, 2020, pág. 219).

La COVID-19 puede producir daño y disfunción miocárdica. De hecho, la elevación de la troponina y las anormalidades electrocardiográficas son hallazgos frecuentes. Un análisis de los pacientes de China subraya la importancia de la lesión cardíaca en la COVID-19 severa en alrededor del 20\% de los pacientes se observó elevación de la troponina I, hsTNI. Aquellos con lesión cardíaca eran en promedio 10

años mayores y tenían mayor prevalencia de hipertensión arterial $(59,8 \%$ vs $23,4 \%$, $\mathrm{p}<0,001)$, diabetes (24,4\% vs $12,0 \%, p$ $=0,008)$, enfermedad coronaria $(29,3 \%$ vs $6,0 \%, p<0,001)$ e insuficiencia cardíaca crónica (14,6 vs 1,5\%, p < 0,001). Sin embargo, el dato más relevante es la fuerte relación entre lesión cardíaca y mortalidad por COVID-19: 51,2\% con lesión vs 4,5\% sin ella $(p<0,001)$. Debe remarcarse que el curso de la enfermedad fue más acelerado ante la presencia de lesión cardíaca (Salazar, Barochiner, Espeche, \& Ennis, 2020, págs. 177-178).

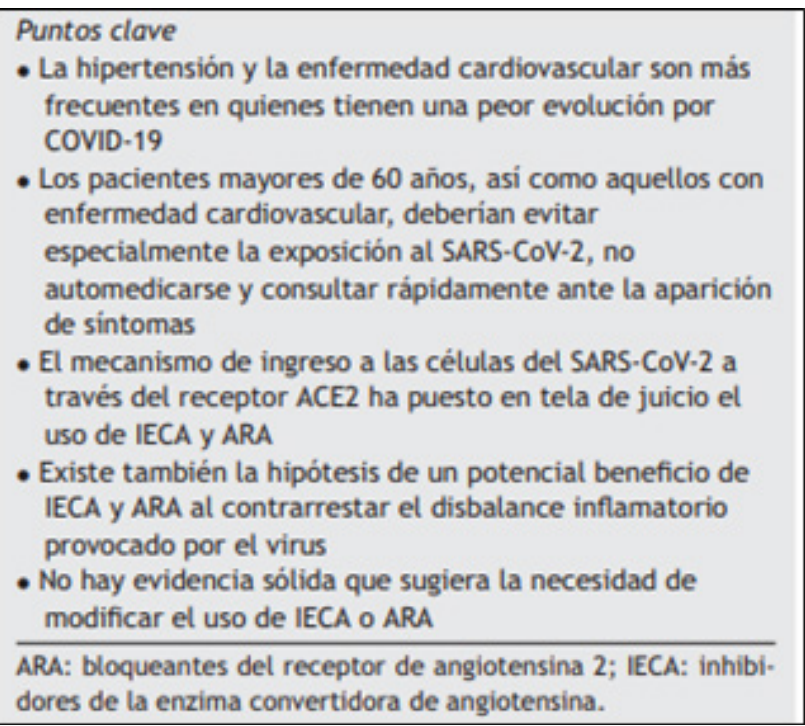

Figura 1. COVID-19 y su relación con la hipertensión y la enfermedad cardiovascular

Fuente: (Salazar, Barochiner, Espeche, \& Ennis, 2020).

El COVID-19 se asocia característicamente a una "tormenta de citoquinas" que desemboca en la inflamación sistémica progresiva, el fallo multiorgánico y la muerte. En este contexto, varios factores se han asociado a la afectación cardiaca, además de la inflamación, fenómenos de trombosis e hipercoagulabilidad, incremento del tono adrenérgico, regulación negativa de los receptores ACE2, o efectos de la medicación. Entre las manifestaciones cardiovasculares de la infección hay que destacar el daño miocárdico agudo y la miocarditis, las arritmias, y fenómenos trombóticos como los síndromes coronarios agudos o la tromboembolia de pulmón (Sánchez, y otros, 2020, pág. 125). 


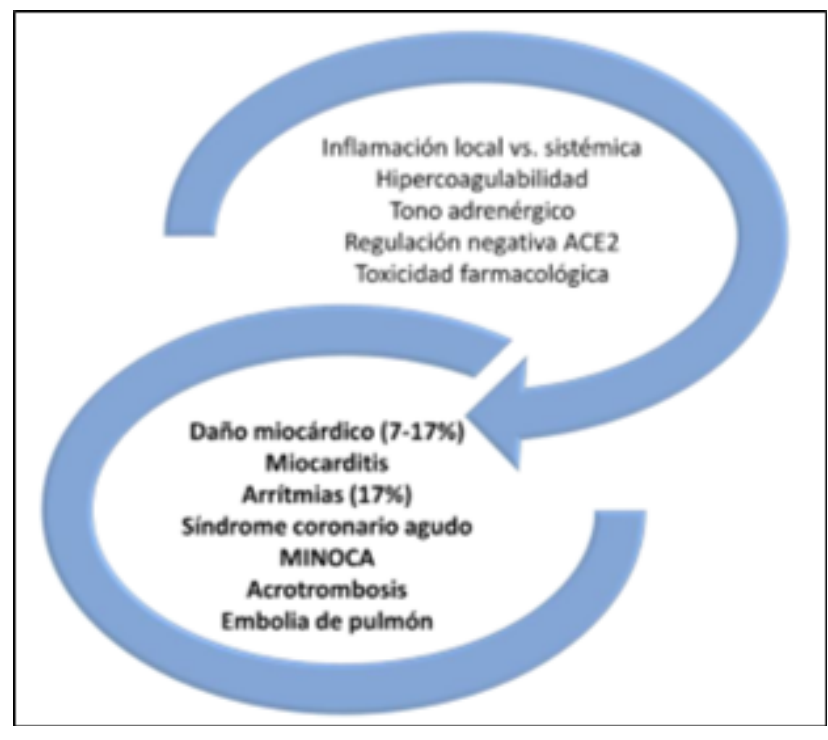

Figura 2. Esquema de mecanismos posibles y manifestaciones cardiovasculares de la infección por SARS-CoV-2. MINOCA: infarto de miocardio sin lesiones coronarias obstructivas.

Fuente: (Sánchez, y otros, 2020)

Los cuadros severos de la enfermedad se asocian con arritmias cardíacas, en especial, los pacientes que desarrollan miocarditis, insuficiencia cardíaca o síndrome coronario agudo. Por tanto, la infección por el virus SARS-CoV-2 que produce COVID-19, es suficiente para desencadenar trastornos del ritmo cardiaco en pacientes sin antecedentes de arritmias, ni cardiopatía estructural como sustrato arritmogénico (Lopez, y otros, 2020, pág. 2). Si bien el mecanismo de la lesión cardíaca no se conoce completamente, se teoriza que SARS-CoV-2 puede provocar afectación cardíaca a través de múltiples mecanismos: 1) daño cardíaco indirecto debido a una respuesta inflamatoria inmune exagerada y tormenta de citoquinas; 2) daño directo mediante la invasión de los cardiomiocitos; y 3) hipoxia severa por daño respiratorio agudo causado por el virus, que puede provocar estrés oxidativo y lesiones miocárdicas por aumento de la demanda miocárdica de oxígeno en presencia de SDRA (Noria, Bachini, \& Ramos, 2020, pág. 222).

La enzima convertidora de angiotensina-2 (ECA-2), receptor crucial del SARS-CoV-2, también se expresa en el corazón, por lo que proporciona el enlace entre los coronavirus y el sistema cardiovascular. La COVID-19, como la influenza estacional, causa una enfermedad leve y autolimitada en la mayoría de las personas infectadas; pero puede ser grave, sobre todo en pacientes mayores o con comorbilidades como: diabetes mellitus, enfermedad pulmonar obstructiva y renal crónicas, entre otras afecciones, donde se incluyen las enfermedades cardiovasculares. Estas pueden volverse inestables en el contexto de la infección viral como consecuencia del desequilibrio entre el aumento de la demanda metabólica inducida por la infección y la reducción de la reserva cardíaca (Moreno-Martínez, Moreno-López, \& Oroz Moreno, 2020, pág. 6).

\section{Metodología}

La metodología empleada en la presente investigación, es una revisión bibliográfica apoyada en medios electrónicos para la obtención primaria de la información, lo que se busca es compendiar la mayor cantidad de investigaciones referentes al caso de estudio, para de esa manera, hacer una síntesis bien precisa que ayude a futuras investigaciones.

\section{Resultados}

\section{A manera general}

Los estudios de necropsia muestran afectaciones graves en los pulmones y el corazón (miocarditis, vasculitis y necrosis de células miocárdicas), y el hígado y la vesícula biliar se encuentran degenerados con necrosis focal e infiltrados con neutrófilos. Lo mismo sucede en los glomérulos y los túbulos renales, donde se hallan microtrombos y focos fibróticos en el intersticio renal. También en el cerebro existe hiperemia cerebral y edema con degeneración de células cerebrales. Se han descrito fibrosis pulmonares residuales, cardiopatías isquémicas, miocardiopatías de origen vírico con insuficiencia cardíaca e incluso casos de miocarditis fulminante de pronóstico gra- 
ve (Choez, Cerón, Zambrano, \& Pin, 2020).

\section{En el ámbito de la cardiología}

1. COVID-19, lesión miocárdica y miocarditis

Casi todos los pacientes hospitalizados muestran niveles elevados de creatina quinasa (CK) y lactato deshidrogenasa (LDH) que sugieren un componente de daño muscular. Adicionalmente, los principales estudios publicados han puesto de manifiesto una elevación significativa de troponina cardiaca en el $7-17 \%$ de los pacientes. Zhou y colaboradores reportaron elevación de troponina I de alta sensibilidad (hs-cT$\mathrm{nl}>28 \mathrm{pg} / \mathrm{mL}$ ) en el $46 \%$ de pacientes que fallecieron, frente a únicamente el $1 \%$ de los pacientes que sobrevivieron al ingreso hospitalario. En el estudio de Guo y cols., la mortalidad de los pacientes con elevación de Troponina T (>99\% del límite superior de la normalidad) osciló entre el $37 \%$ en pacientes sin ECV previa, y el 69\% en pacientes con ECV previa.

Por el contrario, algunos pacientes desarrollarían un cuadro de miocarditis con elevación de la troponina desde los primeros días de ingreso. Aunque aún no se ha realizado de forma sistemática biopsia endomiocárdica para la identificación de partículas virales, una explicación factible sería la infección directa del virus seguida de la respuesta inflamatoria del huésped. Se han comunicado casos de miocarditis fulminante con sintomatología predominantemente cardiaca (shock cardiogénico, dolor torácico sin obstrucción coronaria) y severa afectación de la función ventricular, así como recuperación con inmunoglobulina intravenosa y esteroides a dosis altas (Sánchez, y otros, 2020).

\section{COVID-19 y arritmia cardiaca}

Como se ha observado en otras infecciones virales, tanto la afectación de la función cardiaca como las alteraciones metabólicas y autonómicas pueden favorecer la aparición de arritmias. En el estudio de Wang y cols. Se observó alguna arritmia en 1 de cada 6 pacientes ingresados por COVID-19, en concreto en el $44 \%$ de los que requirieron tratamiento en $\mathrm{UCl}$ y el $7 \%$ de los que permanecieron en planta de hospitalización. Guo y cols. Registraron una incidencia de arritmias ventriculares malignas (taquicardia ventricular o fibrilación ventricular) del $6 \%$, particularmente en pacientes con elevación de troponina cardiaca. Por último, es importante enfatizar el potencial proarrítmico de la medicación específica, que se abordará en detalle más adelante (Sánchez, y otros, 2020).

La infección pulmonar desencadena una respuesta inflamatoria sistémica mediada por linfocitos T y macrófagos. A nivel local produce un síndrome de distrés respiratorio agudo (SDRA) con hipoxemia severa. El proceso inflamatorio, en ocasiones, genera una respuesta exagerada y descontrolada del sistema inmune, que termina con la liberación de gran cantidad de citoquinas (interleucina-6, interleucina-7, interleucina-22 y proteína 10 inducible por interferón). Fenómeno que se conoce como "tormenta de citoquinas" y es responsable de muchas de las complicaciones de la enfermedad (Lopez, y otros, 2020).

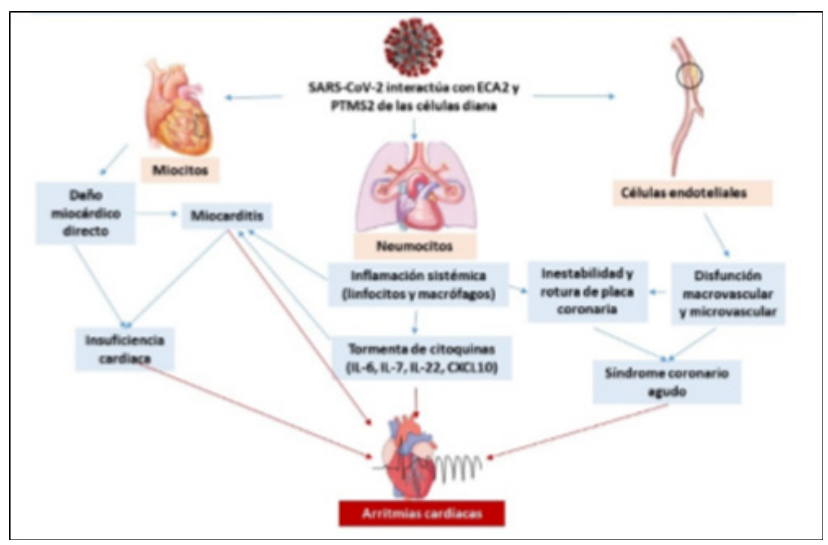

Figura 3. Arritmias cardíacas causada por la COVID19

Fuente: (Lopez, y otros, 2020) 


\section{Síndromes coronarios agudo}

Si bien la enfermedad viral y la inflamación pueden desestabilizar las placas ateroscleróticas, los informes iniciales indican que la mayoría de los casos corresponden a infarto de miocardio sin lesiones coronarias obstructivas (MINOCA del acrónimo en inglés). En una comunicación personal de 34 pacientes tratados en la región de Lombardía, en el 62\% no se identificó una lesión trombóticos culpable. El mecanismo en estos casos podría ser un infarto "tipo 2" (disbalance entre demanda y aporte) o la infección directa de los pericitos con inflamación y trombosis microvascular. Debido a lo anterior, la decisión para el manejo invasivo versus no invasivo de los síndromes coronarios agudos debe considerarse cuidadosamente (Sánchez, y otros, 2020).

\section{Insuficiencia cardíaca descompensada}

La presentación como IC es frecuente, alcanzando cerca de $23 \%$ de los infectados por SARS-CoV-2. Sin embargo, persisten dudas acerca de si se debe más frecuentemente a una exacerbación de patología previa o a un fenómeno de novo, secundario a miocarditis o miocardiopatía por estrés. No es de extrañar que al igual que en otras afecciones respiratorias, los pacientes con SARS-CoV-2 y antecedentes de IC crónica presenten descompensación de la misma. Estos casos se dan generalmente con niveles de troponina en sangre y marcadores de respuesta inflamatoria bajos, lo que hace suponer que el compromiso directo del miocardio no es el principal responsable (Noria, Bachini, \& Ramos, 2020).

5. Efectos crónicos sobre el sistema cardiovascular

El seguimiento a 12 años de los pacientes recuperados de la infección por SARS-CoV reveló anomalías del sistema cardiovascular en el 44\%, y alteraciones lipídicas o glucémicas en el $68 \%$ y $60 \%$ respectivamente. Parece que la infección viral conduce a trastornos del metabolismo de los lípidos y la glucosa por mecanismos aún inciertos. Dado que el SARS-CoV-2 tiene una estructura similar al SARS-CoV, este nuevo virus también podría causar daño crónico al sistema cardiovascular, por lo que se debe enfatizar la prevención del riesgo vascular a largo plazo en estos pacientes (Sánchez, y otros, 2020).

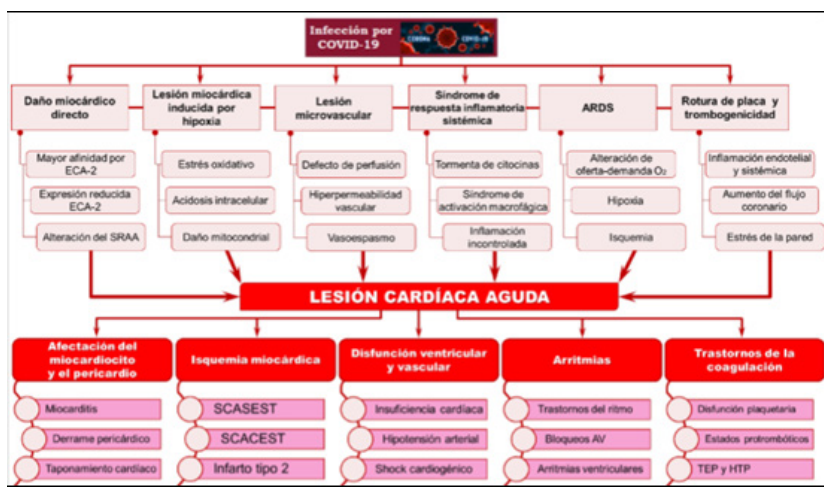

Figura 4. Mecanismos fisiopatológicos de la lesión cardíaca aguda en la COVID-19 y sus principales manifestaciones clínicas

Fuente: (Moreno-Martínez, Moreno-López, \& Oroz Moreno, 2020).

La respuesta a la infección viral produce un estado de hipercoagulabilidad que, unido a la inflamación de las células endoteliales, puede generar disfunción plaquetaria y predisposición a la formación de trombos que, aunque son más frecuentemente venosos, también pueden aparecer en el sistema arterial y producir infartos a cualquier nivel; así como tromboembolia e hipertensión pulmonares. La expresión máxima de este trastorno es la presencia de una coagulopatía similar al síndrome antifosfolípido o el establecimiento de una coagulación intravascular diseminada (Moreno-Martínez, Moreno-López, \& Oroz Moreno, 2020).

\section{Conclusiones}

Está claro que los mayores riesgos de desarrollo de patologías a causas del Covid-19, se pueden presentar en pacientes con enfermedades ya existentes que por el debilitamiento del sistema inmune ataca al organismo, pudiendo agravar aún más las condiciones del paciente, hasta pudiendo ocasionar su muerte. 
El Covid-19 es un virus que ha atacado al sistema inmune de tal manera de deja secuelas que aun en este momento que se redacta este artículo se siguen investigando y siguen apareciendo otras. Dentro de la bibliografía consultada destacan la aparición de patologías cardiacas en muchos de los casos, arritmias, pudiendo generar más afecciones del corazón, ya descritas anteriormente.

A pesar de todo esto, la fisiopatología por infección a causa del Covid-19, el sistema cardiovascular es sumamente importante, en donde los niveles de comorbilidad son altos, asociados a resultados obviamente clínicamente adversos.

En el caso de pacientes que han padecido el Covid-19 de una manera de moderada a severa y que les han dado neumonía, el aumento de la actividad inflamatoria sistémica y procuagulante, puede persistir mucho después del término de la infección y pueden generar mayor riesgo de enfermedad cardiovascular hasta 10 años después.

\section{Bibliografía}

Choez, M. M., Cerón, D., Zambrano, C., \& Pin, V. (2020). Riesgos y consecuencias de los pacientes contagiados con COVID 19. RECIMUNDO, 4(2), 217-225. doi:10.26820/recimundo/4.(2). mayo.2020.217-225

Lopez, A. G., Cardentey, M., Betancourt, A., Chirino, O., López, F., \& Rodríguez, R. (2020). Arritmias cardíacas en pacientes con la COVID-19. Escenarios y tratamiento. Revista Cubana de Cardiología y Cirugía Cardiovascular, 26(3), 1006. Obtenido de http://revcardiologia.sld.cu/index.php/revcardiologia/article/view/1006

Moreno-Martínez, F. L., Moreno-López, F., \& Oroz Moreno, R. (2020). Repercusión cardiovascular de la infección por el nuevo coronavirus SARS-CoV-2 (COVID-19). CorSalud, 12(1), 3-17. Obtenido de http://scielo.sld.cu/scielo.php?pi$d=$ S2078-71702020000100003\&script=sci_arttext\&tIng=en

Noria, S., Bachini, J., \& Ramos, M. (2020). Coronavirus y sistema cardiovascular. Revista Uruguaya de Cardiología, 35(2), 193-208. doi:http://dx.doi. org/10.29277/cardio.35.2.13

Salazar, M., Barochiner, J., Espeche, W., \& Ennis, I. (2020). COVID-19, hipertensión y enfermedad cardiovascular. Hipertensión y riesgo vascular, 37(4), 176-180. doi:https://doi.org/10.1016/j.hipert.2020.06.003

Sánchez, D. M., Pascual, C., Marschal, A., Calva, F., Muñoz, M., \& Antón, S. (2020). Aspectos cardiológicos relevantes en la infección COVID-19. Revista de Investigación y Educación en Ciencias de la Salud (RIECS), 5(1), 121-133. doi:https://doi. org/10.37536/RIECS.2020.5.1.203

\section{CITAR ESTE ARTICULO:}

Moyon Uriña, S. J., Villacís Nieto, J. M., Giler Navia, D. X., \& Castro Caicedo, K. Y. (2020). Cardiopatías asociadas al COVID-19. RECIAMUC, 4(4), 248-254. https://doi.org/10.26820/reciamuc/4.(4).noviembre.2020.248-254 